\title{
COMMON FIXED POINT THEOREMS BY ALTERING DISTANCES
}

\author{
K. JHA AND R. P. PANT
}

\begin{abstract}
In this paper we obtain common fixed point theorems for weakly commuting pairs of self mappings by altering distances between the points under a $\phi$-contractive condition.
\end{abstract}

\section{Introduction}

The study of common fixed points for self mappings on a metric space by altering distances between the points with the use of control functions has emerged as an area of wide interest. Khan et al. [2] established fixed point theorem for a single self map. Sastry and Babu [7] proved fixed point theorem for a pair of self maps. Sastry et al. [8] proved a unique common fixed point theorem for four mappings by using a control function in order to alter distances between the points. Pant et al. [5, 6] obtained an answer to the open problem of Sastry et al. [8] by establishing a connection between continuity and reciprocal continuity in the setting of control function.

The presence of control function creates certain difficulties in proving the existence of fixed point under contractive conditions. In view of these difficulties, known fixed-point theorems either employ a stronger contractive condition like the Banach contractive condition e.g. in Sastry et al. [8] or assume the existence of a convergent sequence of iterates e.g. in [2], [7]. The study of fixed points in the presence of control function under more general contractive conditions like Mier-Keeler type $(\varepsilon, \delta)$-contractive condition or a $\phi$-contractive condition is still an open area. In the present paper, we prove a common fixed point theorem assuming a $\phi$-contractive condition. We employ a control function that unifies the choice of control function in [7], [8]. Also, in the settings of our theorem, we consider the open problem of [8] and provide an answer to the problem in the setting of a more general contractive condition than in Sastry et al. [8].

We have used the following notions.

Definition 1.1. A control function $\psi$ is defined as $\psi: \Re_{+} \rightarrow \Re_{+}$which is continuous, monotonically increasing, $\psi(2 t) \leq 2 \psi(t)$ and $\psi(t)=0$ if and only if $t=0$.

Received September 3, 2002; revised February 19, 2003.

2000 Mathematics Subject Classification. 47H10.

Key words and phrases. Fixed points, self maps, compatible maps, reciprocally continuous maps, control function. 
Definition 1.2. Two self mappings $A$ and $S$ of a metric space $(X, d)$ are called weakly commuting if $d(A S x, S A x) \leq d(A x, S x)$ for each $x$ in $X$. This condition implies that $A S x=S A x$ whenever $A x=S x$.

Clearly, commuting and weakly commuting mappings are compatible, but the converses are not necessarily true [1].

Definition 1.3. ([8]) Two self mappings $A$ and $S$ of a metric space $(X, d)$ are called $\psi$-compatible if $\lim _{n} \psi\left(d\left(A S x_{n}, S A x_{n}\right)\right)=0$ whenever $\left\{x_{n}\right\}$ is a sequence such that $\lim _{n} A x_{n}=\lim _{n} S x_{n}=t$ for some $t$ in $X$.

Definition 1.4. ([3]) Two self mappings $A$ and $S$ of a metric space $(X, d)$ are said to be reciprocally continuous in $X$, if $\lim _{n} A S x_{n}=A t$ and $\lim _{n} S A x_{n}=S t$ whenever $\left\{x_{n}\right\}$ is a sequence such that $\lim _{n} A x_{n}=\lim _{n} S x_{n}=t$ for some $t$ in $X$.

Notation 1.5. If $A, B, S$ and $T$ are four self mappings of $(X, d)$ and $\psi$ is a control function on $\Re_{+}$, we write

$$
\begin{gathered}
M_{\psi}(x, y)=\max \{\psi(d(S x, T y)), \psi(d(A x, S x)), \psi(d(B y, T y)), \\
[\psi(d(A x, T y))+\psi(d(S x, B y))] / 2\} .
\end{gathered}
$$

\section{Main Theorem}

Theorem 2.1. Let $(A, S)$ and $(B, T)$ be weakly commuting pairs of self mappings of a complete metric space $(X, d)$ and $\psi$ be as in Definition 1.1 satisfying

(i) $A X \subset T X, B X \subset S X$ and

(ii) $\psi(d(A x, B y)) \leq \phi\left(M_{\psi}(x, y)\right)$, for all $x, y$ in $X$ whenever $M_{\psi}(x, y)>0$ and $\phi: \Re_{+} \rightarrow \Re_{+}$be an upper semi continuous function such that $\phi(t)<t$ for each $t>0$.

Suppose that $(A, S)$ and $(B, T)$ are $\psi$-compatible pairs of reciprocally continuous mappings. Then $A, B, S$ and $T$ have a unique common fixed point.

Proof. Let $x_{0}$ be any point in $X$. Define sequences $\left\{x_{n}\right\}$ and $\left\{y_{n}\right\}$ in $X$ such that

$$
y_{2 n}=A x_{2 n}=T x_{2 n+1} ; \quad y_{2 n+1}=B x_{2 n+1}=S x_{2 n+2} .
$$

We claim that $\left\{y_{n}\right\}$ is a Cauchy sequence. We write $\alpha_{n}=\psi\left(d\left(y_{n}, y_{n+1}\right)\right)$. Then, using condition (ii), it follows that

$$
\begin{aligned}
\alpha_{2 n} & =\psi\left(d\left(y_{2 n}, y_{2 n+1}\right)\right)=\psi\left(d\left(A x_{2 n}, B x_{2 n+1}\right)\right) \\
& \leq \phi\left(M_{\psi}\left(x_{2 n}, x_{2 n+1}\right)\right) \\
& =\phi\left(\operatorname { m a x } \left\{\psi\left(d\left(S x_{2 n}, T x_{2 n+1}\right)\right), \psi\left(d\left(A x_{2 n}, S x_{2 n}\right)\right), \psi\left(d\left(B x_{2 n+1}, T x_{2 n+1}\right)\right),\right.\right. \\
& \left.\left.\quad\left[\psi\left(d\left(S x_{2 n}, B x_{2 n+1}\right)\right)\right] / 2\right\}\right) . \\
& =\phi\left(\operatorname { m a x } \left\{\psi\left(d\left(y_{2 n}, y_{2 n+1}\right)\right), \psi\left(d\left(y_{2 n-1}, y_{2 n}\right)\right), \psi\left(d\left(y_{2 n+1}, y_{2 n}\right)\right),\right.\right.
\end{aligned}
$$




$$
\begin{aligned}
& \left.\left.\quad\left[\psi\left(\max \left\{d\left(y_{2 n}, y_{2 n+1}\right), d\left(y_{2 n}, y_{2 n-1}\right)\right\}\right)\right] / 2\right\}\right) \\
& =\phi\left(\psi\left(d\left(y_{2 n-1}, y_{2 n}\right)\right)\right) \\
& \leq \phi\left(\psi\left(d\left(y_{2 n-1}, y_{2 n}\right)\right)\right)=\phi\left(\alpha_{2 n-1}\right) .
\end{aligned}
$$

That is,

$$
\alpha_{2 n} \leq \phi\left(\alpha_{2 n-1}\right)<\alpha_{2 n-1}
$$

Similarly, $\alpha_{2 n-1}<\alpha_{2 n-2} ; \alpha_{2 n-2}<\alpha_{2 n-3}$ and so on. Thus $\left\{\alpha_{n}\right\}=\left\{\psi\left(d\left(y_{n}, y_{n+1}\right)\right)\right\}$ is a strictly decreasing sequence of positive numbers and hence converges, say, to $\alpha \geq 0$. Suppose $\alpha>0$. Then the inequality (2.1.2) on making $n \rightarrow \infty$ and in view of upper semi continuity of $\phi$ yields $\alpha \leq \phi(\alpha)<\alpha$, a contradiction. Hence $\alpha=\lim _{n \rightarrow \infty} \psi\left(d\left(y_{n}, y_{n+1}\right)\right)=0$. This, by the monotonically increasing property of $\psi$, implies

$$
\lim _{n \rightarrow \infty} d\left(y_{n}, y_{n+1}\right)=0,
$$

and also $\left\{d\left(y_{n}, y_{n+1}\right)\right\}$ is a strictly decreasing sequence of positive numbers. We now show that $\left\{y_{n}\right\}$ is a Cauchy sequence.

Suppose it is not. Then there exists an $\varepsilon>0$ and a subsequence $\left\{y_{n_{i}}\right\}$ of $\left\{y_{n}\right\}$ such that $d\left(y_{n_{i}}, y_{n_{i}+1}\right)>2 \varepsilon$. But since $\lim _{n \rightarrow \infty} d\left(y_{n}, y_{n+1}\right)=0$, there exists an integer $m_{i}$ satisfying $n_{i}<m_{i}<n_{i+1}$ such that $d\left(y_{n_{i}}, y_{m_{i}}\right) \geq \varepsilon$. If not, then

$$
d\left(y_{n_{i}}, y_{n_{i+1}}\right) \leq d\left(y_{n_{i}}, y_{n_{i+1}-1}\right)+d\left(y_{n_{i+1}}, y_{n_{i+1}-1}\right)<\varepsilon+d\left(y_{n_{i+1}}, y_{n_{i+1}-1}\right)<2 \varepsilon,
$$

a contradiction. If $m_{i}$ is the smallest integer such taht $d\left(y_{n_{i}}, y_{m_{i}}\right) \geq \varepsilon$, then

$$
\begin{aligned}
\varepsilon & \leq d\left(y_{n_{i}}, y_{m_{i}}\right) \leq d\left(y_{n_{i}}, y_{m_{i}-2}\right)+d\left(y_{m_{i}-2}, y_{m_{i}-1}\right)+d\left(y_{m_{i}-1}, y_{m_{i}}\right) \\
& <\varepsilon+d\left(y_{m_{i}-2}, y_{m_{i}-1}\right)+d\left(y_{m_{i}-1}, y_{m_{i}}\right) .
\end{aligned}
$$

That is, there corresponds an integer $m_{i}$ satisfying $n_{i}<m_{i}<n_{i+1}$ such that

$$
d\left(y_{n_{i}}, y_{m_{i}}\right) \geq \varepsilon \quad \text { and } \quad \lim _{n_{i} \rightarrow \infty} d\left(y_{n_{i}}, y_{m_{i}}\right)=\varepsilon
$$

From the triangle inequality, we get $\left|d\left(y_{n_{i}}, y_{m_{i}+1}\right)-d\left(y_{n_{i}}, y_{m_{i}}\right)\right| \leq d\left(y_{m_{i}+1}, y_{m_{i}}\right)$. Thus, as $n_{i} \rightarrow \infty$, we obtain $d\left(y_{m_{i}+1}, y_{n_{i}}\right) \rightarrow \varepsilon$. Similarly, we can get $d\left(y_{n_{i}+1}, y_{m_{i}}\right) \rightarrow \varepsilon$. Applying the continuity of $\psi$, we get either

$$
\psi\left(d\left(y_{m_{i}+1}, y_{n_{i}}\right)\right) \rightarrow \psi(\varepsilon) \quad \text { or } \quad \psi\left(d\left(y_{n_{i}+1}, y_{n_{i}}\right)\right) \rightarrow \psi(\varepsilon) .
$$

Moreover, $m_{i}$ can be chosen in such a manner that $m_{i}$ is even, when $n_{i}$ is odd and $m_{i}$ is odd, when $n_{i}$ is even. Suppose that $n_{i}$ is odd and $m_{i}$ is even. Then by virtue of (ii), we get

$$
\psi\left(d\left(y_{n_{i}+1}, y_{m_{i}+1}\right)\right)=\psi\left(d\left(A x_{n_{i}+1}, B x_{m_{i}+1}\right)\right) \leq \phi\left(M_{\psi}\left(x_{n_{i}+1}, x_{m_{i}+1}\right)\right) .
$$

On letting $n_{i} \rightarrow \infty$ and in view of result (2.1.5) and applying the upper semi continuity of $\phi$, the above inequality yields $\psi(\varepsilon) \leq \phi(\psi(\varepsilon))<\psi(\varepsilon)$, a contradiction. Hence $\left\{y_{n}\right\}$ is 
a Cauchy sequence. Since $X$ is complete, there is a point $z$ in $X$ such that $y_{n} \rightarrow z$ as $n \rightarrow \infty$. Hence from (2.1.1), we have

$$
y_{2 n}=A x_{2 n+1}=T x_{2 n+1} \rightarrow z \quad \text { and } \quad y_{2 n+1}=B x_{2 n+1}=S x_{2 n+2} \rightarrow z
$$

Now suppose that $(A, S)$ is a $\psi$-compatible pair of reciprocally continuous mappings. Since $A$ and $S$ are reciprocally continuous, by (2.1.6), we get

$$
A S x_{2 n} \rightarrow A z \quad \text { and } \quad S A x_{2 n} \rightarrow S z
$$

Also, $\psi$-compatibility of $A$ and $S$ implies that $\lim _{n} \psi\left(d\left(A S x_{2 n}, S A x_{2 n}\right)\right)=0$. We now show that $A z=S z$.

Suppose $A z \neq S z$. Let $\varepsilon=(d(A z, S z)) / 2$. Then there exists $N$ in $Z^{+}$such that $\psi\left(d\left(A S x_{2 n}, S A x_{2 n}\right)\right)<\psi(\varepsilon)$ for all $n \geq N$. This implies that $d\left(A S x_{2 n}, S A x_{2 n}\right)<\varepsilon$ for all $n \geq N$. Hence by $(2.1 .7), d(A z, S z)<\varepsilon=(d(A z, S z)) / 2$, a contradiction.

Hence

$$
A z=S z
$$

Since $A X \subset T X$, there is a point $w$ in $X$ such that $T w=A z$. By (2.1.8),

$$
T w=A z=S z
$$

Now, we show that $A z=B w$. Suppose $A z \neq B w$. Then, by (ii), we have

$$
\psi(d(A z, B w)) \leq \phi\left(M_{\psi}(z, w)\right) \leq \phi(\psi(d(B w, T w)))<\psi(d(B w, A z)),
$$

a contradiction. Hence $A z=B w$. Therefore, by (2.1.9),

$$
B w=A z=S z=T w .
$$

Since $A$ and $S$ are weakly commuting, we have by (2.1.10),

$$
A S z=S A z \quad \text { and } \quad A A z=A S z=S A z=S S z .
$$

Since $B$ and $T$ are weakly commuting, we have

$$
B B w=B T w=T B w=T T w .
$$

We now show that $A A z=A z$. Suppose $A A z \neq A z$. Then by (ii), we get

$$
\begin{aligned}
\psi(d(A z, A A z)) & =\psi(d(B w, A A z)) \\
& \leq \phi(M \psi(A z, w)) \\
& =\phi(\psi(d(A z, A A z))), \quad(\text { by }(2.1 .10) \&(2.1 .12))
\end{aligned}
$$

a contradiction. Hence $A A z=A z$. 
Also, we have $A A z=S A z$. Therefore, $A z$ is a common fixed point for $A$ and $S$. Also, suppose $B B w \neq B w$. By (ii), we have

$$
\begin{aligned}
\psi(d(B w, B B w)) & =\psi(d(A z, B B w)) \quad(\text { by }(2.1 .10)) \\
& \leq \phi(M \psi(z, B w)) \\
& =\phi(\psi(d(B w, B B w))), \quad(\text { by }(2.1 .10) \&(2.1 .12)) \\
& <\psi(d(B w, B B w)),
\end{aligned}
$$

a contradiction. Hence $B B w=B w$ and since $T B w=B B w$, we have $B w$ as a common fixed point for $B$ and $T$. Since $A z=B w$, we have $A z$ as a common fixed point for $A, B$, $S$ and $T$. Uniqueness of a common fixed point follows by (ii). The proof is similar when the pair $(B, T)$ is assumed $\psi$-compatible and reciprocally continuous. This completes the proof of the theorem.

In the above theorem, we replace reciprocal continuity of $B$ and $T$ by continuity of $A$ and obtain result similar to Theorem 2.1,

Theorem 2.2. Let $(A, S)$ and $(B, T)$ be weakly commuting pairs of self mappings of a complete metric space $(X, d)$ and $\psi$ be as in definition (1.1) satisfying

(i) $A X \subset T X, B X \subset S X$ and

(ii) $\psi(d(A x, B y)) \leq \phi\left(M_{\psi}(x, y)\right)$, for all $x, y$ in $X$ whenever $M_{\psi}(x, y)>0$ and $\phi$ : $\Re_{+} \rightarrow \Re_{+}$be an upper semi continuous function such that $\phi(t)<t$ for each $t>0$.

Suppose that $A$ and $S$ are $\psi$-compatible and $A$ is continuous mapping. Then $A, B$, $S$ and $T$ have a unique common fixed point.

Proof. Let $x_{0}$ be any fixed point in $X$. Define sequences $\left\{x_{n}\right\}$ and $\left\{y_{n}\right\}$ in $X$ given by the rule

$$
y_{2 n}=A x_{2 n}=T x_{2 n+1} \quad \text { and } \quad y_{2 n+1}=B x_{2 n+1}=S x_{2 n+2} .
$$

This can be done by virtue of (i). Then applying the same proof as that in the Theorem 2.1 , we can show that $\left\{y_{n}\right\}$ is a Cauchy seuqence. Since $X$ is a complete metric space, there is a point $z$ in $X$ such that

$$
y_{2 n}=A x_{2 n}=T x_{2 n+1} \rightarrow z \quad \text { and } \quad y_{2 n+1}=B x_{2 n+1}=S x_{2 n+2} \rightarrow z .
$$

Now, suppose that $(A, S)$ is $\psi$-compatible then we have

$$
A x_{2 n} \rightarrow z \quad \text { and } \quad S x_{2 n} \rightarrow z \quad \text { implies that } \lim _{n} \psi\left(d\left(A S x_{2 n}, S A x_{2 n}\right)\right)=0 .
$$

Also, since $A$ is continuous, so by (2.2.2), we get

$$
A A x_{2 n} \rightarrow A z \quad \text { and } \quad A S x_{2 n} \rightarrow A z \quad \text { as } n \rightarrow \infty \text {. }
$$

We claim that $\lim _{n} S A x_{2 n}=A z$. Using (2.2.3), we get

$$
\psi\left(d\left(S A x_{2 n}, A z\right)\right) \leq \psi\left(d\left(S A x_{2 n}, A S x_{2 n}\right)+d\left(A S x_{2 n}, A z\right)\right) \rightarrow 0 \quad \text { as } n \rightarrow \infty .
$$


Thus, we get $d\left(S A x_{2 n}, A z\right) \rightarrow 0$ as $n \rightarrow \infty$, and so $\lim _{n} S A x_{2 n}=A z$. Also, since $A X \subset T X$, for each $n$, there exists $w_{2 n}$ in $X$ such that $A A x_{2 n}=T w_{2 n}$ and $A A x_{2 n}=$ $T w_{2 n} \rightarrow A z$. Thus, $A A x_{2 n} \rightarrow A z, S A x_{2 n} \rightarrow A z, A S x_{2 n} \rightarrow A z$ and $T w_{2 n} \rightarrow A z$ as $n \rightarrow \infty$. Again, we claim that $\lim _{n} B w_{2 n} \rightarrow A z$. If not, then there exist $\varepsilon>0$ and a subsequence $\left\{n_{k}\right\}$ such that $d\left(A A x_{2 n_{k}}, B w_{2 n_{k}}\right)>\varepsilon$ and $\psi\left(d\left(S A x_{2 n_{k}}, A S x_{2 n_{k}}\right)\right)<\varepsilon$ for all $n_{k}$. Therefore,

$$
\begin{aligned}
\psi(\varepsilon) \leq & \psi\left(d\left(A A x_{2 n_{k}}, B w_{2 n_{k}}\right)\right) \\
\leq & \phi\left(M_{\psi}\left(A x_{2 n_{k}}, w_{2 n_{k}}\right)\right) \\
= & \phi\left(\operatorname { m a x } \left\{\psi\left(d\left(S A x_{2 n_{k}}, T w_{2 n_{k}}\right)\right), \psi\left(d\left(A A x_{2 n_{k}}, S A x_{2 n_{k}}\right)\right), \psi\left(d\left(B w_{2 n_{k}}, T w_{2 n_{k}}\right)\right),\right.\right. \\
& \left.\left.\quad\left[\psi\left(d\left(A A x_{2 n_{k}}, T w_{2 n_{k}}\right)\right)+\psi\left(d\left(B w_{2 n_{k}}, S A x_{2 n_{k}}\right)\right)\right] / 2\right\}\right) \\
= & \phi\left(\max \left\{\psi\left(d\left(B w_{2 n_{k}}, T w_{2 n_{k}}\right)\right),\left[\psi\left(d\left(B w_{2 n_{k}}, S A x_{2 n_{k}}\right)\right)\right] / 2\right\}\right), \\
= & \phi\left(\psi\left(d\left(B w_{2 n_{k}}, A A x_{2 n_{k}}\right)\right)\right), \\
& <\psi\left(d\left(B w_{2 n_{k}}, A A x_{2 n_{k}}\right)\right), \quad \text { a contradiction. }
\end{aligned}
$$

Hence $\lim _{n} B w_{2 n}=A z$.

We claim that $A z=S z$. For this, using (ii), we get

$$
\begin{aligned}
\psi\left(d\left(S z, B w_{2 n}\right)\right) \leq & \phi\left(M_{\psi}\left(z, w_{2 n}\right)\right) \\
= & \phi\left(\operatorname { m a x } \left\{\psi\left(d\left(S z, T w_{2 n}\right)\right), \psi(d(A z, S z)), \psi\left(d\left(B w_{2 n}, T w_{2 n}\right)\right),\right.\right. \\
& \left.\left.\quad\left[\psi\left(d\left(A z, T w_{2 n}\right)\right)+\psi\left(d\left(B z, S w_{2 n}\right)\right)\right] / 2\right\}\right), \\
= & \phi\left(\max \left\{\psi\left(d\left(S z, T w_{2 n}\right)\right), \psi(d(A z, S z)),\left[\psi\left(d\left(S z, B w_{2 n}\right)\right)\right] / 2\right\}\right) .
\end{aligned}
$$

Letting $n \rightarrow \infty$, we get

$$
\begin{aligned}
\psi(d(S z, A z)) & \leq \phi(\max \{\psi(d(S z, A z))),[\psi(d(S z, A z))] / 2\}) \\
& =\phi(\psi(d(S z, A z))), \quad \text { a contradiction. }
\end{aligned}
$$

Thus we have

$$
A z=S z .
$$

Since $A X \subset T X$, there exists some $w$ in $X$ such that $A z=T w$. Therefore, we have

$$
A z=S z=T w .
$$

Moreover, we show that $A z=B w$. Suppose on the contrary that $A z \neq B w$. Then, using (ii), we get

$$
\begin{aligned}
\psi(d(A z, B w)) \leq & \phi\left(M_{\psi}(z, w)\right) \\
= & \phi(\max \{\psi(d(S z, T w)), \psi(d(A z, S z)), \psi(d(B w, T w)), \\
& \quad[\psi(d(A z, T w))+\psi(d(B z, S w))] / 2\}), \\
= & \phi(\max \{\psi(d(B w, A z)),[\psi(d(B w, A z))] / 2\}), \\
= & \phi(\psi(d(B w, A z))), \quad \text { a contradiction. }
\end{aligned}
$$


Therefore, $A z=B w$. Hence

$$
A z=S z=T w=B w .
$$

Since $A$ and $S$ are weakly commuting, we have by (2.2.7), $A S z=S A z$ and hence

$$
A A z=A S z=S A z=S S z
$$

and by the weakly commuting property of $B$ and $T$, we get

$$
B B w=B T w=T B w=T T w .
$$

We now show that $A A z=A z$. Suppose that $A A z \neq A z$ then by (ii), we get

$$
\psi(d(A z, A A z))=\psi(d(B w, A A z)) \leq \phi\left(M_{\psi}(A z, w)\right)=\phi(\psi(d(A z, A A z))),
$$

(using (2.2.7) \& (2.2.8)), a contradiction. Hence $A A z=A z$. Also, we have $A A z=S A z$. Therefore, $A z$ is a common fixed point of $A$ and $S$. Again, suppose that $B B w \neq B w$. Then using (ii), we get

$$
\begin{aligned}
\psi(d(B w, B B w)) & =\psi(d(A z, B B w)) \quad(\text { by }(2.2 .6)) \\
& \leq \phi\left(M_{\psi}(z, B w)\right) \\
& =\phi(\psi(d(B w, B B w))), \quad \text { (by using }(2.2 .7) \&(2.2 .9)) \\
& <\psi(d(B w, B B w)), \quad \text { a contradiction. }
\end{aligned}
$$

Hence $B B w=B w$ and since $T B w=B B w$, we have $B w$ being a common fixed point for $B$ and $T$. Finally, since $A z=B w$, we have $A z$ as a common fixed point for $A, B, S$ and $T$. Moreover, the uniqueness of a common fixed point follows from (ii). This completes the proof of the theorem.

Remark. The proof is similar when the pair $(A, S)$ is assumed $\psi$-compatible and $S$ is continuous. Moreover, we can get the same result when the $(B, T)$ is assumed $\psi$-compatible and either $T$ or $B$ is assumed to be continuous.

The following example shows that if $A$ and $S$ are not continuous in Theorem 2.2 then the result of Theorem 2.2 is not true. That is, all the mappings $A, B, S$ and $T$ do not have common fixed point.

Example 2.3. Let $X=[0,1]$ with the Euclidean metric $d$. Define $A=B$ and $S=T: X \rightarrow X$ by the rule $A 0=1 / 2, A x=x / 4$ for $0<x \leq 1$ and $S 0=1, S x=x / 2$ for $0<x \leq 1$. Then $A$ and $S$ are weakly commuting mappings and hence they are $\psi$ compatible, with $\psi$ being an identity mapping. Also, $A$ and $S$ satisfy both the conditions (i) and (ii) of Theorem 2.2 with $\phi(t)=t / 2$. But $A$ and $S$ are not continuous and they do not have common fixed point.

\section{Acknowledgement}

The authors take this opportunity to express their sincere thanks to an anonymous referee for valuable comments. 


\section{References}

[1] S. M. Kang and B. E. Rhoades, Fixed points for four mappings, Math. Japonica 37(1992), 1053-1059.

[2] M. S. Khan, M. Swaleh and S. Sessa, Fixed point theorem by altering distances between the points, Bull. Austral. Math. Soc. 30(1984), 1-9.

[3] R. P. Pant, Common fixed points of four mappings, Bull. Cal. Math. Soc. 90(1998), 281286.

[4] R. P. Pant, A common fixed point theorem under a new condition, Indian J. Pure Appl. Math. 30(1999), 147-152.

[5] R. P. Pant, K. Jha and A. B. Lohani, A note on common fixed points by altering distances, Tamkang J. Math. (2003) (to appear).

[6] R. P. Pant, K. Jha and S. Padaliya, On common fixed points by altering distances between the points, Tamkang J. Math. (2003) (to appear).

[7] K. P. R. Sastry and G. V. R. Babu, Some fixed point theorems by altering distances between the points, Ind. J. Pure and Appl. Math. 30(June 1999), 641-647.

[8] K. P. R. Sastry, S. V. R. Naidu, G. V. R. Babu and G. A. Naidu, Generalization of common fixed point theorems for weakly commuting mappings by altering distances, Tamkang Journal of Math. 31(2000), 243-250.

Department of Mathematical Sciences, Kathmandu University, P.O.Box 6250, Kathmandu, Nepal.

E-mail: jhaknh@yahoo.co.in

Department of Mathematics, Kumaon University, D.S.B. Campus, Nainital-263002, Uttaranchal, India. 